\title{
Production of oligosaccharides and cellobionic acid by Fibrobacter succinogenes S85 growing on sugars, cellulose and wheat straw Régis Nouaille $^{1,2}$, Maria Matulova ${ }^{1,3}$, Vladimír Pätoprstý ${ }^{3}$, Anne-Marie Delort ${ }^{1}$ and Evelyne Forano ${ }^{2}$
}

(1) Laboratoire de Synthèse et Etude de Systèmes à Intérêt Biologique, UMR 6504 Université Blaise Pascal-CNRS, 63177 Aubière cedex, France

(2) Unité de Microbiologie UR454, Centre de Recherches de Clermont-Ferrand-Theix, INRA, 63122 Saint-Genès-Champanelle, France

(3) Institute of Chemistry, Center for Glycomics, Slovak Academy of Sciences, Dubravska cesta 9, 842 38 Bratislava, Slovak Republic

Abstract : Extracellular culture fluid of Fibrobacter succinogenes S85 grown on glucose, cellobiose, cellulose or wheat straw was analysed by 2D-NMR spectroscopy. Cellodextrins did not accumulate in the culture medium of cells grown on cellulose or straw. Maltodextrins and maltodextrin- $1 P$ were identified in the culture medium of glucose, cellobiose and cellulose grown cells. New glucose derivatives were identified in the culture fluid under all the substrate conditions. In particular, a compound identified as cellobionic acid accumulated at high levels in the medium of $F$. succinogenes $\mathrm{S} 85$ cultures. The production of cellobionic acid (and cellobionolactone also identified) was very surprising in an anaerobic bacterium. The results suggest metabolic shifts when cells were growing on solid substrate cellulose or straw compared to soluble sugars.

Keywords: Rumen, Fibrobacter succinogenes, Cellobionate, NMR, Oligosaccharides

\section{Introduction :}

Cellulose is the most abundant biomass in the world, and its biological degradation is a key step in global carbon cycling as well as a promising approach for the production of bioenergy (Lynd et al. 2002). The rumen ecosystem, by exploiting cellulolytic microorganisms, is the most efficient process for cellulose transformation into useful products. The rumen cellulolytic microorganisms, which possess very active and complex hydrolytic systems, are thus potential biocatalysts for the synthesis of highly added value products. Fibrobacter succinogenes S85 is recognised as one of the most active cellulolytic rumen bacteria. Its enzymatic system has been extensively studied by molecular and biochemical approaches, and many different cellulases and hemicellulases have been identified (Krause et al. 2003). In addition, the complete genome sequence of the strain S85 of $F$. succinogenes revealed more than 100 predicted enzymes active against plant polysaccharide (Qi et al. 2005), suggesting a high potential hydrolytic activity of this organism. The sugar metabolism by $F$. succinogenes $\mathrm{S} 85$ was also extensively investigated (Forano et al. 2008), while metabolisation of natural substrates was much less explored.

In a previous work, we showed that resting cells of $F$. succinogenes $\mathrm{S} 85$ were able to synthesise and release oligosaccharides identified by 2D-NMR techniques as maltodextrins (MD), maltodextrin-1phosphate (MD-1P) and another unknown phosphorylated sugar. These metabolites were produced when cells were metabolising glucose and cellobiose, but also their natural substrate cellulose (Matulova et al. 2001; Nouaille et al. 2004, 2005). The synthesis and excretion of MD and MD-1P were unexpected in bacteria specialised in cellulose degradation. In addition, we found that no cellodextrins were accumulating in extracellular media of cells whatever the substrate and particularly cellulose, contrary to what was generally admitted. These results prompted us to investigate whether growing bacteria were still producing these oligosaccharides. Extracellular culture fluids of bacteria growing on soluble sugars and also on cellulose and wheat straw were analysed by 2D-NMR spectroscopy for the presence of $\alpha(1-4)$ and $\beta(1-4)$ glucose oligosaccharides, phosphorylated or not. Analysis of sugar metabolism and its regulation in $F$. succinogenes growing on natural substrate is a prerequisite for further attempts to improve lignocellulose bioconversion by this species.

\section{Materials and methods :}

Experimental procedures were mostly as described in Nouaille et al. (2004) or Matulova et al. (2005). Bacterial growth 
F. succinogenes S85 (ATCC 19169) was grown at $39^{\circ} \mathrm{C}$ for $10 \mathrm{~h}$ in an anaerobic chemically defined medium (Gaudet et al. 1992) with $8 \mathrm{~g} \mathrm{~L}^{-1}$ glucose or cellobiose or for 4 days with $10 \mathrm{~g} \mathrm{~L}^{-1}$ of Sigmacell 20 cellulose or ground wheat straw: dried Triticum sp. straw was first chopped up to a length particle of $5-10 \mathrm{~mm}$ and then ground to particles $<500 \mu \mathrm{m}$ (Waring laboratory blender, VWR International, Fontenay-sous-Bois, France). Cellulose and wheat straw cultures (in triplicate) were harvested after 1, 2 or 4 days of growth. Extracellular media were separated from cells by centrifugation (15 min at 20 , $000 \times g$ ) before analysis.

\section{NMR experiments}

After centrifugation, $\mathrm{pH}$ of cell-free supernatants was corrected to 7.40, and the supernatants were freezedried two times with $\mathrm{D}_{2} \mathrm{O}$. Samples were finally dissolved in a mixture of $470 \mu \mathrm{L} 99.98 \% \mathrm{D}_{2} \mathrm{O}, 20 \mu \mathrm{L}$ $10 \mathrm{mM}$ sodium 3-(trimethylsilyl) propionate $\mathrm{TSP}_{4} \mathrm{~d}_{4}\left(\begin{array}{ll}\delta & 0.0\end{array}\right)$ and $10 \mu \mathrm{L} 50 \mathrm{mM}$ 1-O-methyl- $\beta$-Dxylopyranose $(\delta 4.331 / 104.79)$ used as standards. Samples were subjected to liquid state NMR measurements at $300 \mathrm{~K}$ on Bruker Avance DSX $300 \mathrm{MHz}$ and $500 \mathrm{MHz}$ spectrometers in $5 \mathrm{~mm}$ TXI inverse probes $\left({ }^{1} \mathrm{H},{ }^{13} \mathrm{C},{ }^{15} \mathrm{~N}\right)$ and Varian $600 \mathrm{MHz}$ UNITY INOVA $600 \mathrm{NB}$ spectrometer in $5 \mathrm{~mm}$ ${ }^{1} \mathrm{H}\left\{{ }^{13} \mathrm{C}\right.$, $\left.{ }^{15} \mathrm{~N}\right\}$ PFG Triple Res IDTG600-5 probe, respectively, all with z-gradients. The following techniques were used for the assignment of NMR signals: one-dimensional transient gradient-enhanced nuclear overhauser enhancement spectroscopy (ge-NOESY), ge-1D TOCSY (gradient-enhanced onedimensional total-correlation spectroscopy), two-dimensional gradient enhanced proton-homonuclear shift correlation spectroscopy (ge-COSY), ${ }^{1} \mathrm{H}-{ }^{13} \mathrm{C}$ gradient-enhanced heteronuclear single quantum coherence spectroscopy (ge-HSQC) and ${ }^{1} \mathrm{H}-{ }^{13} \mathrm{C}$ heteronuclear multiple quantum correlation spectroscopy (HMBC). Standards were dissolved in an anaerobic buffer $(50 \mathrm{mM}$ potassium phosphate, $40 \mathrm{mM}$ $\mathrm{Na}_{2} \mathrm{CO}_{3}, 3 \mathrm{mM}$ cysteine) at $\mathrm{pH} 7.4$; they were freeze-dried and dissolved in $\mathrm{D}_{2} \mathrm{O}$.

\section{Metabolite assays}

Succinate and acetate were quantified using enzymatic assays (Roche Diagnostics, Meylan, France). Cultures were done in triplicate. Standard error was lower than $12 \%$.

\section{Chemicals}

TSP- $\mathrm{d}_{4}$ was purchased from Eurisotop (Saint-Aubin, France). Glucose, cellobiose, Sigmacell 20 cellulose and 1-O-methyl- $\beta$-D-xylopyranose were from Sigma-Aldrich (Saint-Quentin Fallavier, France). All enzymes and other chemicals were purchased from Sigma-Aldrich or Roche.

\section{Results}

\section{Growth of F. succinogenes S85 on different substrates}

Growth of F. succinogenes S85 on glucose, cellobiose, cellulose and wheat straw was monitored by quantification of the production of succinate and acetate, the two main products of its metabolism. Identical growth was obtained on glucose and cellobiose, and the same amounts of succinate $(8.4 \pm 1.0 \mathrm{mM})$ and acetate $(2.3 \pm 0.8 \mathrm{mM})$ were produced in the extracellular medium after $10 \mathrm{~h}$ (stationary phase, not shown). When cells were grown on cellulose and wheat straw, respectively, 10.5 and $7 \mathrm{mM}$ of succinate and 8 and $4 \mathrm{mM}$ of acetate were produced after 4 days (stationary phase), on the example shown in Fig. 1. Both on cellulose and wheat straw, acetate was produced in higher amount than succinate during the first $24 \mathrm{~h}$, while after this time, succinate was the major metabolite produced (Fig. 1). Cultures on glucose and cellobiose always produced succinate in higher concentration than acetate (mean ratio about 3.5). There appears thus to be a metabolic shift after the first day of culture on solid substrate. 


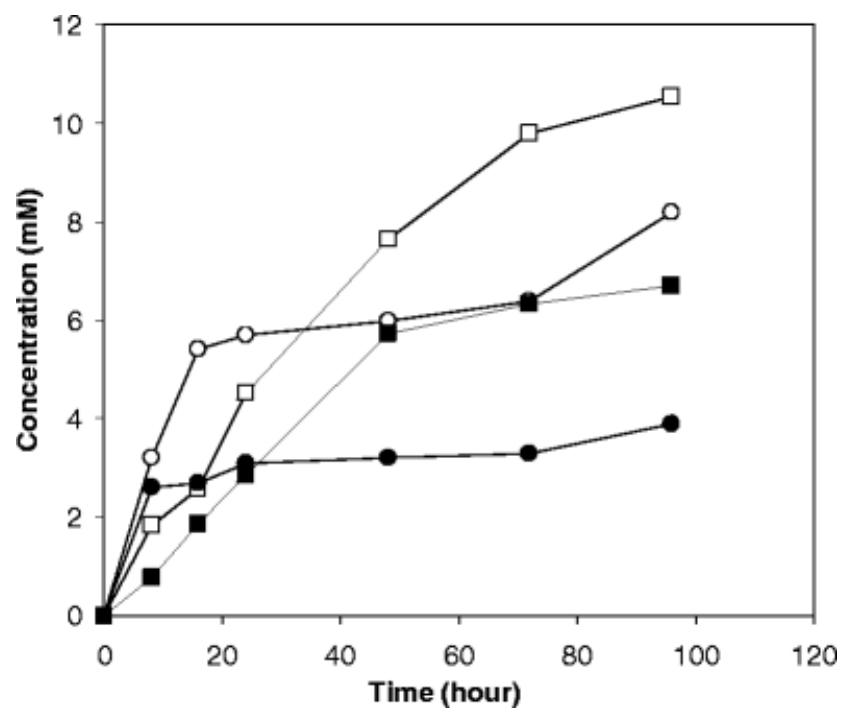

Fig. 1 Production of succinate (squares) and acetate (circles) by F. succinogenes $\mathrm{S} 85$ growing on cellulose (open symbols) or wheat straw (closed symbols). Cells were cultivated for 4 days in mineral medium with $10 \mathrm{~g} \mathrm{~L}^{-1}$ Sigmacell 20 cellulose or wheat straw. Metabolites were quantified by enzymatic assays. Standard error was less than $12 \%$

\section{Analysis of glucose, cellobiose and cellulose culture media}

${ }^{1} \mathrm{H}$ NMR COSY spectra of extracellular media obtained after $10 \mathrm{~h}$ growth on glucose or cellobiose and 1 day growth on cellulose are presented in Fig. 2 as an example. The left-hand part of Fig. 2 shows spectra of the region of the H1/H2 cross-peaks of non-substituted MD ( $\delta 5.41 / 3.59$ ppm, Fig. 2a, b), MD$1 P(\delta 5.46 / 3.52$ and 5.41/3.59, Fig. 2c) and of the unknown compound $\mathrm{X}$ (glucose-1P-derivative, $5.46 / 3.49$, Fig. 2a, b and c). These metabolites were previously observed in resting cell incubations (Nouaille et al. 2005). The signals of $\alpha$-glucose were also present in this part of the figure, corresponding to free glucose ( $\alpha$-glucose, $\alpha$ Glc, $\delta 5.24 / 3.54$, Fig. 2a and 2b) or corresponding to residual cellobiose $(\alpha$ reducing end, $\alpha \mathrm{CB} \delta 5.24 / 3.59$, Fig. 2 b). In the right part of Fig. 2 are detected the signals of free glucose ( $\beta$-glucose, $\beta$ Glc, $\delta 4.65 / 3.24$, Fig. $2 a$ ) and cellobiose ( $\beta$ reducing end, $\beta C B, \delta 4.68 / 3.30$ and non-reducing glucose moiety of CB, $\beta 4.52 / 3.33$, Fig. $2 \mathrm{a}$ and $\mathrm{b}$ ). The presence of cross-peaks at $\delta 4.54 / 3.34$ suggested the synthesis of CD of $d p>2$ (Fig. 2b). A huge signal of a new metabolite, named X2 $(\delta 4.63 / 3.39)$ was detected in Fig. 2a, b and c. 

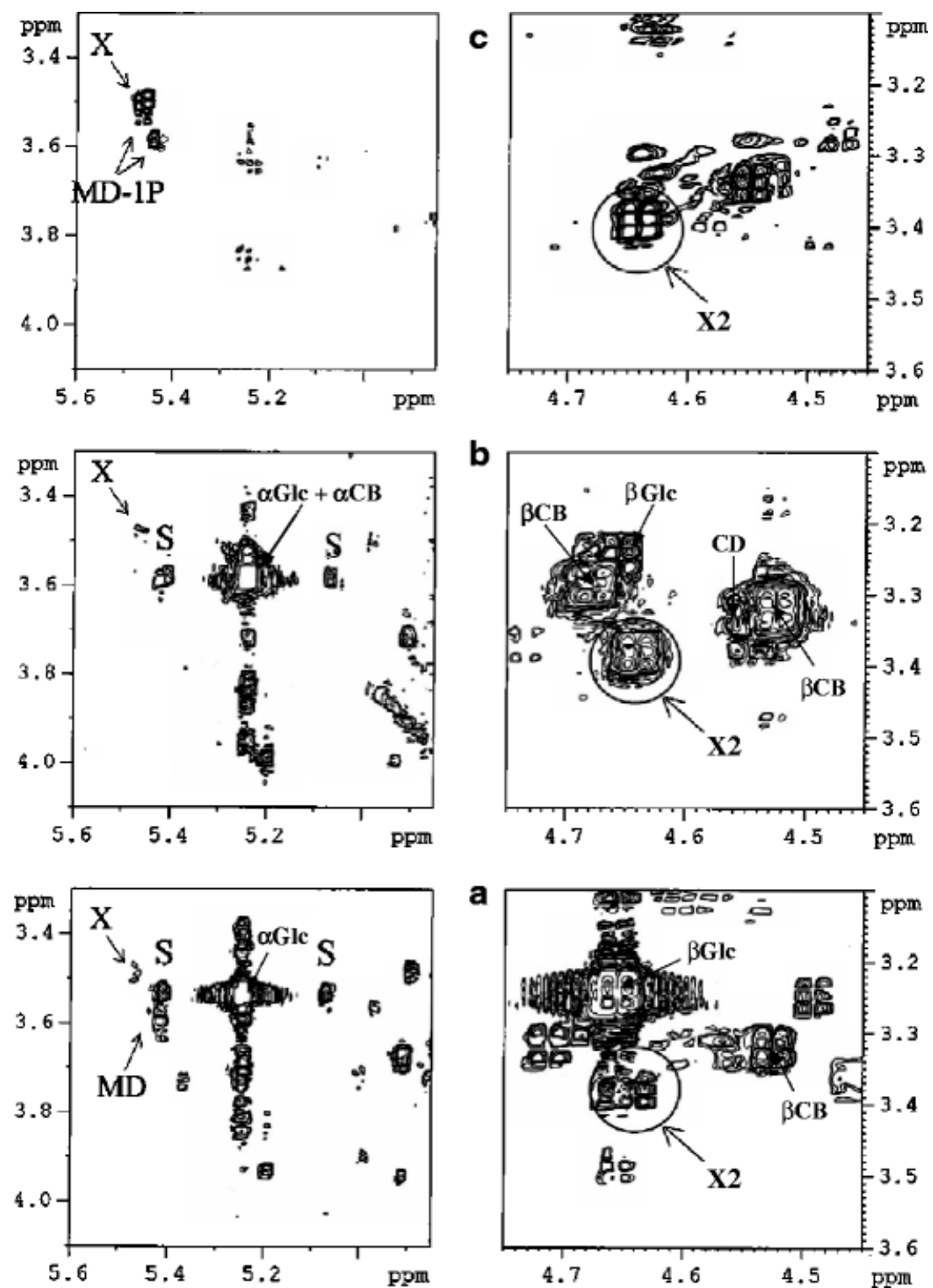

Fig. 2 Part of ${ }^{1} \mathrm{H}-{ }^{1} \mathrm{H}$ COSY NMR spectra of culture fluid on glucose, cellobiose and cellulose. F. succinogenes cells were grown for $10 \mathrm{~h}$ on glucose (a) or cellobiose (b) or for 1 day on cellulose Sigmacell 20 (c). Glc glucose, $C B$ cellobiose, $C D$ cellodextrins, $M D$ linear maltodextrins, $M D-1 P$ maltodextrin-1-phosphate, $X$ glucose-1P derivative, $X 2$ unknown glucose derivative, $S$ side bands

\section{Identification of X2}

Various 1D and 2D-NMR techniques were used to analyse the cellobiose culture media in order to identify X2. The COSY spectrum revealed the presence of glucose and X2 both in a rough ratio 1:1 and a further series of non-identified signals. Starting with selective excitation of the signal at $\delta 4.63$, series of 1D selective TOCSY, 1D selective NOESY were obtained. Two-dimensional HSQC and HMBC NMR spectra were also registered (Fig. 3). NMR data indicated the presence of disaccharide in which a nonreducing part of the molecule is $\beta$-glucose, which is linked to an acyclic carbon chain. The type of linkages between the two sugar moieties was confirmed by responses in the $1 \mathrm{D}{ }^{1} \mathrm{H}$ transient NOESY spectra. After a selective excitation of $\mathrm{H} 1$ at $\delta 4.63$ (glucose of non-reducing end), besides its own $\mathrm{H} 3$ ( $\delta$ $3.52)$ and $\mathrm{H} 5(\delta 3.49)$, two other signals at $\delta 4.21$ and 4.13 appeared, which were previously identified by 1D selective TOCSY as H4 and H3 signals of the acyclic moiety (Table 1). In the HMBC spectrum, a cross-peak due to $\mathrm{H} 2 / \mathrm{C} 1$ of acyclic carbon chain was observed, confirming thus the presence of carboxyl group at $\mathrm{C} 1$ (Fig. 3b). These data suggest that X2 is cellobionic acid in form of cellobionate (CBA-S, cellobionic acid salt). NMR spectra of cellobionate and cellobionolactone were previously published by Higham et al. 1994. Our data (Table 1, Fig. 4) are in very good agreement with the spectrum of CBA-S shown there. Moreover, the published spectrum of cellobionolactone (CBA-L) helped us to identify further signals in our spectra and to reveal the presence of this compound in the $F$. succinogenes culture medium (Fig. 4). Anomeric signal due to non-reducing glucose unit of CBA-L was found at $\delta$ 4.54. 
Reducing end glucose unit in the form of lactone showed a typical signal at $\delta 4.27$ in the ${ }^{1} \mathrm{H}$ NMR spectrum (Fig. 4). However, HSQC spectrum (Fig. 3a) indicated that it represents overlapped signals of three protons of the lactonic part of the molecule. Their assignment was done only tentatively by comparison with the data of glucose, gluconolactone and cellobiose. The ratio of signals due to cellobiose/glucose/CBA-S/CBA-L was 0.1:1:1:0.6 in the spectrum shown. The quantification in the case of CB, CBA-S and CBA-L was done by integration of $\mathrm{H} 1$ signal due to non-reducing end units and $\mathrm{H} 1 \alpha$ and $\beta$ signals of reducing-end glucose. The ratio CBA-S/CBA-L varied from 1 to 1.7 depending on the experiments.
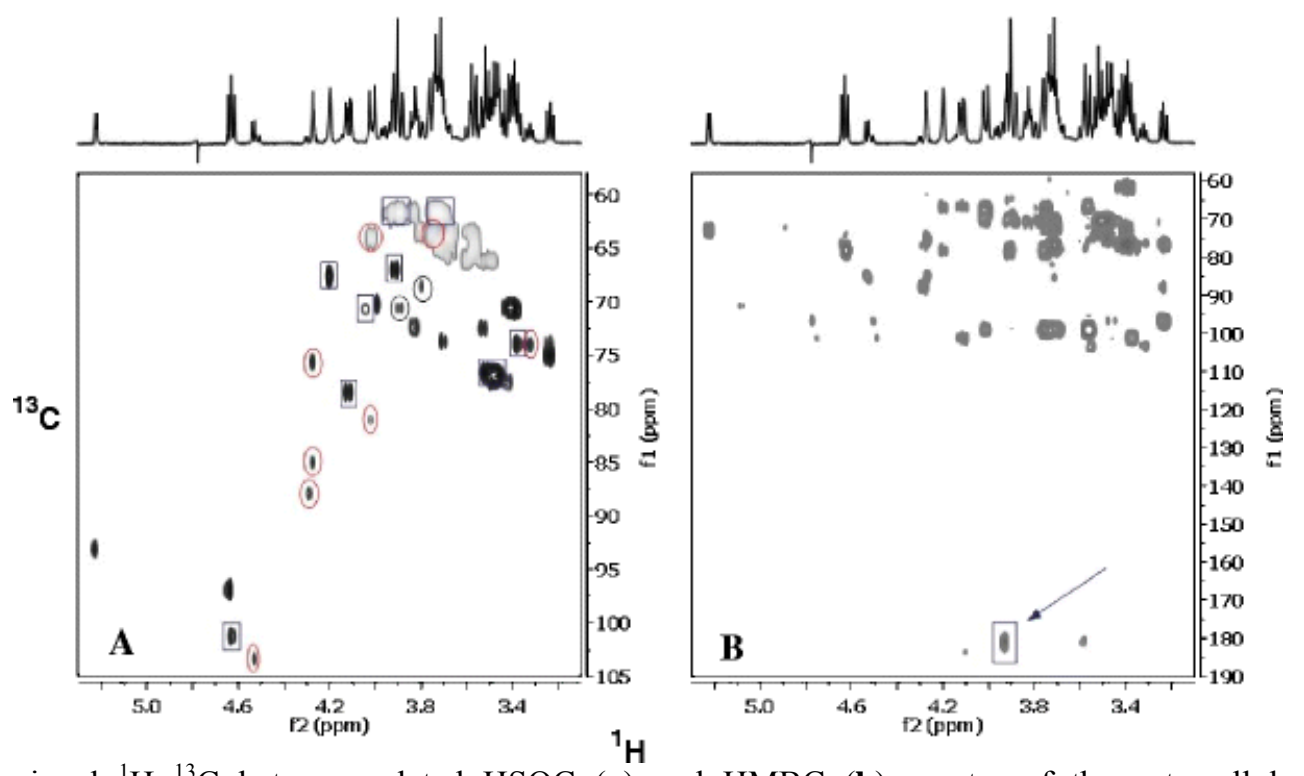

Fig. 3 Two-dimensional ${ }^{1} \mathrm{H}^{13}{ }^{13}$ heterocorrelated HSQC (a) and HMBC (b) spectra of the extracellular medium of $F$. succinogenes grown on cellobiose. Spectrum A Cross-peaks marked by squares are due to cellobionic acid in sodium salt form (CBA-S), while those marked by circles are due to a cellobionolactone (CBA-L). Other signals present in the spectrum are due to $\alpha$ and $\beta$ glucose (H1 signals at $\delta 5.23 / 93.1$ and 4.65/96.9, respectively) formed by degradation of cellobiose. H1 signal at $\delta$ 4.51 reveals the presence of a trace amount of cellobiose still present in the mixture. Arrow in the spectrum $B$ indicates a crosspeak due to a long range coupling constant from $\mathrm{H} 2$ to carboxyl group in the lactone part of the molecule in CBA-S

Table $1{ }^{1} \mathrm{H}$ and ${ }^{13} \mathrm{C}$ NMR chemical shifts of cellobionate and cellobionolactone

\begin{tabular}{|c|c|c|c|c|c|c|c|}
\hline & \multirow[t]{2}{*}{ Sugar unit } & \multicolumn{6}{|c|}{ Chemical shift $\delta / \mathrm{ppm}$} \\
\hline & & $\mathrm{H} 1 / \mathrm{C} 1$ & $\mathrm{H} 2 / \mathrm{C} 2$ & $\mathrm{H} 3 / \mathrm{C} 3$ & $\mathrm{H} 4 / \mathrm{C} 4$ & $\mathrm{H} 5 / \mathrm{C} 5$ & $\mathrm{H} 6, \mathrm{H}^{\prime} / \mathrm{C} 6$ \\
\hline \multirow[t]{2}{*}{ CBA-S } & $\operatorname{Glc}(1 \rightarrow 4)$ & $4.63 / 101.2$ & $3.39 / 73.9$ & $3.52 / 76.7$ & $3.46 / 76.7$ & $3.49 / 76.8$ & $\begin{array}{l}3.91 \\
3.73 / 61.8\end{array}$ \\
\hline & $\begin{array}{l}\mathrm{COO}^{-}- \\
(\mathrm{CHOH})_{5}-\mathrm{CH}_{2} \mathrm{OH}\end{array}$ & 182.2 & $3.92 / 66.9$ & $4.13 / 78.3$ & $4.21 / 67.6$ & $4.05 / 70.1$ & $\begin{array}{l}4.02, \\
3.76 / 64.1\end{array}$ \\
\hline \multirow[t]{2}{*}{ CBA-L* } & $\operatorname{Glc}(1 \rightarrow 4)$ & $4.54 / 103.3$ & $3.34 / 73.9$ & $3.52 / 76.9$ & $3.41 / 70.6$ & $3.50 / 76.8$ & $\begin{array}{l}3.95 \\
3.73 / 61.8\end{array}$ \\
\hline & Glc-lactone & - & $4.29 / 85.1$ & $4.29 / 75.6$ & $4.30 / 87.8$ & $4.04 / 81.0$ & $\begin{array}{l}3.72, \\
3.57 / 65.4\end{array}$ \\
\hline
\end{tabular}

Assignment of CBA-L was done only tentatively by comparison of its chemical shifts with those due to glucose, gluconolactone and cellobiose 


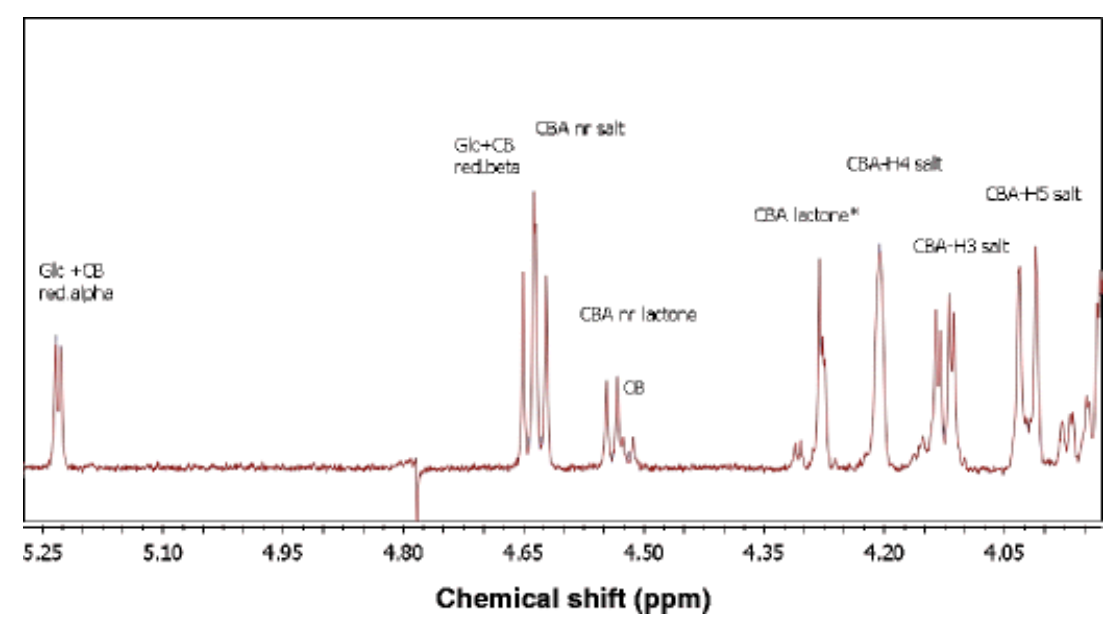

Fig. 4 Part of the ${ }^{1} \mathrm{H}$ spectrum of the extracellular medium of $F$. succinogenes grown on cellobiose. Glc $+C B$ red.alpha overlapped H1 signals due to $\alpha$-glucose and reducing $\alpha$-glucose units of cellobiose, Glc $+C B$ red.beta overlapped H1 signals due to $\beta$-glucose and reducing $\beta$-glucose units of cellobiose, $C B A n r$ salt $\mathrm{H} 1$ signal due to non-reducing unit glucose of cellobionate, $C B A \mathrm{nr}$ lactone $\mathrm{H} 1$ signal due to non-reducing unit glucose of cellobionolactone, $C B \mathrm{H} 1$ signal due to nonreducing unit glucose of cellobiose, $C B A$ lactone* tentatively attributed overlapped $\mathrm{H} 2$, H3 and H4 signals of the gluconolactone part of the cellobionolactone molecule, $C B A-H 4$ salt $\mathrm{H} 4$ of the acyclic part of the cellobionate molecule, $C B A$ $H 3$ salt $\mathrm{H} 3$ of the acyclic part of the cellobionate molecule, $C B A-H 5$ salt $\mathrm{H} 5$ of the acyclic part of the cellobionate molecule

\section{Analysis of wheat straw culture media :}

We also checked for the presence of these compounds in the culture media of bacteria grown on wheat straw. Samples were analysed by ${ }^{1} \mathrm{H}-{ }^{1} \mathrm{H}$ COSY. Spectra collected after 1 and 4 days of bacterial growth are presented in Fig. $\underline{5}$. Identified signals were mainly due to free xylose, arabinose and arabinoglucuronoxylan oligosaccharides, released during hemicellulose degradation in wheat straw (Matulova et al. 2005). The H1 anomeric signal due to non-reducing Glc unit of cellobionate (CBA-S, $\mathrm{H} 1 / \mathrm{H} 2: \delta$ 4.63/3.38) was also detected in the COSY spectra from the beginning of bacterial growth (Fig. 5a) until the end of the culture (Fig. 5b). Its concentration, quantified from H1/H2 signal volumes in COSY spectra, varied from 1 to $1.5 \mathrm{mM}$. The presence of cellodextrin cross-peaks $(\delta 4.53-4.52 / 3.36-$ 3.32) was not detected unambiguously in any spectrum because a lot of $\mathrm{H} 1 / \mathrm{H} 2$ cross-peaks were detected in the region $\delta 4.60-4.50 / 3.35-3.30$. However, as shown previously in similar experiments and also complementary experiments (Matulova et al. 2005), cellodextrins are likely not present in the straw culture medium. The metabolite denoted $\mathrm{X}(\mathrm{H} 1 / \mathrm{H} 2$ and $\mathrm{C} 1: \delta 5.46 / 3.48$ and 94.2$)$ and assigned to a derivative of Glc-1P was detected in the early stage of the culture (after $8 \mathrm{~h}$, not shown), but it was not found in the spectra registered at later stages (Fig. 5). MD and MP-1P were not identified in the wheat straw culture media.
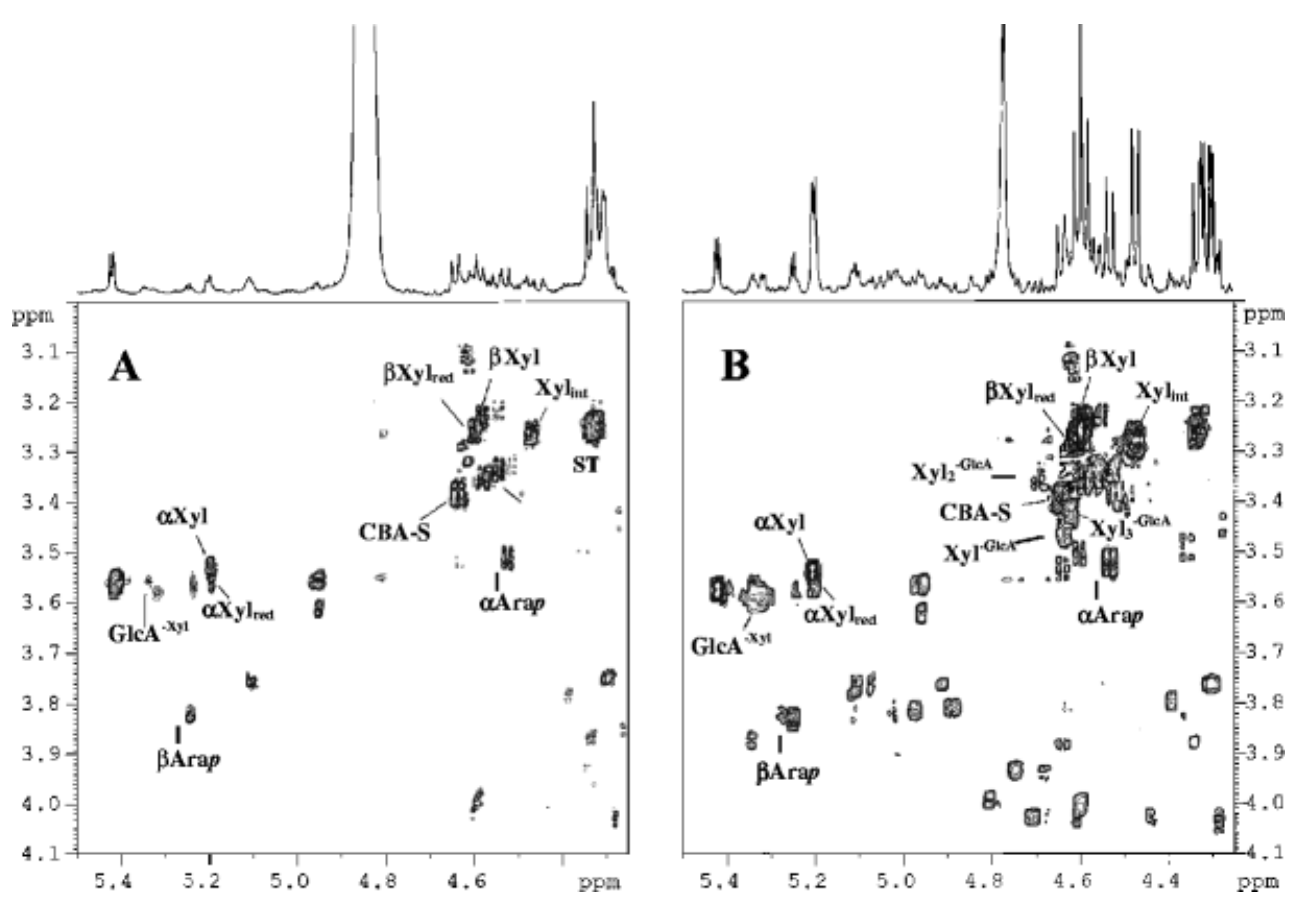
Fig. $5{ }^{1} \mathrm{H}-{ }^{1} \mathrm{H}$ COSY NMR spectra of culture media collected after 1 day (a) and 4 days (b) during the degradation of wheat straw by $F$. succinogenes S85. GlcA ${ }^{-X y l}$ glucuronic moiety in $x y l o$-oligosaccharides, $X y l^{-G l c A}$ xylose substituted by glucuronic acid in xylo-oligosaccharides, Arap free arabinose in the pyranose form ( $\alpha$ and $\beta$ anomers), $X y l$ free xylose ( $\alpha$ and $\beta$ anomers), $X y l_{\text {red }}$ reducing end of xylose ( $\alpha$ and $\beta$ anomers) in xylooligosaccharides, $X y l_{\text {int }}$ internal xylose in $x y l o$-oligosaccharides, Araf ${ }^{-X y l}$ arabinosyl moiety in $x y l o$-oligosaccharides, $X 2$ cellobionate, $S T$ standard, $1-O$-methyl- $\beta$-D-xylopyranose

\section{Oligosaccharide production in growing cells :}

The presence of different sugars detected by NMR in the extracellular medium of the four culture conditions is summarised in Table 2. The first important result is that neither CD nor cellobiose is accumulating in the extracellular medium of cells growing on cellulose or straw. Also, no glucose could be detected when cells were grown on solid substrate. Maltose- $1 P$ was only found when cellulose was the growth substrate, while non-phosphorylated MDs were only produced when cells were growing on the soluble sugars glucose and cellobiose. The Glc-1P derivative $(\mathrm{X})$ was found under all conditions of growth, while Glc-1P could not be detected on any substrate. Finally, cellobionic acid (cellobionate) was detected under the four conditions of growth; its concentration was in the millimolar range. This concentration value indicates that this compound, as well as the other ones detected in the extracellular medium, was not released from cell lysis (Wells and Russell 1996), but was actively excreted from the bacteria. The presence of all the sugars and sugar derivatives detected previously in resting cells (Nouaille et al. 2005) is also reported in Table $\underline{2}$ for comparison.

Table 2 Detection of different sugars in the extracellular culture fluid of $F$. succinogenes S85 grown on glucose, cellobiose, cellulose and wheat straw (growing cells) or in the extracellular incubation medium of cells incubated with glucose, cellobiose and cellulose (resting cells)

\begin{tabular}{|l|l|l|l|l|l|l|l|l|l|}
\hline Substrate & $\begin{array}{l}\text { Experimental } \\
\text { conditions }\end{array}$ & MD-1P & MD & X & Glc & CB & CD & Glc-1P & CBA \\
\hline Glucose & Growing cells & - & + & + & + & + & - & - & + \\
\hline & Resting cells & + & + & - & + & - & - & + & - \\
\hline Cellobiose & Growing cells & - & + & + & + & + & + & - & + \\
\hline & Resting cells & + & + & - & + & - & - & + & - \\
\hline Cellulose & Growing cells & + & - & + & - & - & - & - & + \\
\hline & Resting cells & + & + & + & + & - & - & + & - \\
\hline Straw & Growing cells & - & - & + Transient & - & - & - & - & + \\
\hline
\end{tabular}

$M D$ - $1 P$ maltose-1-phosphate, $M D$ linear maltodextrins, $X$ glucose-1 $P$ derivative, $G l c$ glucose, $C B$ cellobiose, $C D$ cellodextrins of $\mathrm{dp}>2, C B A$ cellobionic acid

\section{Discussion :}

On cellulose and wheat straw, the production of organic acids by $F$. succinogenes $\mathrm{S} 85$, a good indicator of the bacterial growth (Fields et al. 2000), was very similar (Fig. 1). However, the concentration of both acetate and succinate was lower on straw, probably because of less available substrate for growth. Indeed, although $F$. succinogenes S85 degrades very efficiently the hemicelluloses of straw and particularly xylans, it is not able to metabolise the pentoses or xylooligosaccharides released (Matulova et al. 2005) and only uses glucose and cellobiose for growth (Matte et al. 1992; Matheron et al. 1997). On both solid substrates, a shift in the production of the metabolites acetate and succinate was observed after about $24 \mathrm{~h}$, contrary to what was found on glucose or cellobiose. This reflects a modification in the fluxes in the two corresponding metabolic pathways. As the acetate pathway is an efficient means of producing ATP (Miller 1978; Matheron et al. 1997), this shift might be due to different energy requirements between the beginning and the end of the batch culture on solid substrates. For example, there could be a need of more energy in the first hours of growth on solid substrate for induction of synthesis of specific proteins involved in adhesion (Roger et al. 1990) or cellulolysis (Béra-Maillet et al. 2000). In addition, acetate and succinate production may be affected differently by several factors such as $\mathrm{pH}$, as shown previously on continuous cultures of S85 (Weimer 1993) or on other bacteria (Desvaux 2001). As F. succinogenes also produces formate as a minor metabolite (Miller 1978; Matheron et al. 1997), it would have been interesting to monitor its concentration during the growth on the two substrates to see the evolution of its production with time.

The analysis of the oligosaccharides produced in the culture medium on the four substrates shows specific features. 
MD or MD-1P that were produced by resting cells of $F$. succinogenes (Nouaille et al. 2004, 2005) were also found under more physiological conditions of cells growing on cellulose. They were not detected on straw, either because they are not produced at all or because of a too small concentration on this substrate that also provided lower growth (Fig. 1). We previously observed with resting cells (Nouaille et al. 2005) that the ratio MD-1P/MD was increasing with the DP of the substrate (glucose $<$ cellobiose $<<$ cellulose). This phenomenon appears amplified in growing cells where MD-1P was only detected with cellulose, while MD was only detected with glucose and cellobiose (Table 2). One of the main difference between growing and resting cells concerns Glc-1P and $\mathrm{X}$ : Glc-1P was present in resting cells, and its concentration was increasing with the size of the substrate (Table 2, Nouaille et al. 2005), while it was absent in growing cells. On the contrary, $\mathrm{X}$ was not detected in resting cells metabolising glucose and cellobiose, while it was present in high quantity in cells metabolising cellulose; in growing cells, $\mathrm{X}$ was found under the four substrate conditions, it appears in higher quantity in extracellular medium of cells grown on cellulose (Fig. 2). These results suggest that Glc-1P might be the precursor of $\mathrm{X}$. In the case of cellulose, the larger accumulation of $\mathrm{X}$ (both in growing and in resting cells) could be explained by a higher Glc-1P production. Growth on cellulose or straw differs from that on soluble sugars by several factors: (a) the necessity for bacteria first to adhere to the substrate and second to degrade it, (b) different growth rate, (c) different physiological state of the bacteria; the carbon flow is consequently different in cellulose and/or wheat straw grown cells. The metabolism of another anaerobic cellulolytic bacterium Clostridium cellulolyticum was studied in details in batch and continuous cultures on cellobiose or cellulose, and a modification of metabolic fluxes was also observed between the two substrates (Desvaux et al. 2000; Desvaux et al. 2001).

Accumulation of phosphorylated sugars in the extracellular medium of growing bacteria (present work) but also of resting cells (Nouaille et al. 2004, 2005) is quite surprising. Their estimated concentration in the millimolar range exclude their release by bacterial cell lysis as this would imply an extremely high intracellular concentration, far above that previously measured in F. succinogenes (Matheron et al. 1998). They could be actively excreted from the cells by a specific permease, such as that reported to occur for G6P in Escherichia coli (Van der Zee et al. 1996).

In F. succinogenes resting cells, no $\mathrm{CB}$ or $\mathrm{CD}$ was detected in the extracellular medium of cells incubated with glucose or cellulose. On the contrary, cells growing on glucose accumulated CB in the culture fluid, and cells growing on cellobiose accumulated $\mathrm{CB}$ and $\mathrm{CD}$ in the culture medium; these results are in accordance with those of Wells et al. (1995). However, in cellulose cultures, no CB or CD could be detected in the medium. The same results were observed on wheat straw cultures (present work and Matulova et al. 2005). This suggests that CD are utilised by the cells as soon as they are released during cellulose degradation. This result is in agreement with the concept that the depolymerisation of insoluble cellulose to soluble cellodextrins limits the cellulose fermentation, as proposed by Pavlostathis et al. (1988) for Ruminococcus albus. Such mechanism could avoid a feed-back inhibition of cellulases by cellodextrins. In addition, we did not find any glucose accumulation in the milieu of cells grown on cellulose, contrary to what was found previously in cellulose-fed continuous cultures of $F$. succinogenes and particularly at low $\mathrm{pH}$ (Weimer 1993). This might be explained by different growth rate in batch and continuous cultures.

Several groups showed previously that $F$. succinogenes could be co-cultured with non-cellulolytic bacteria on cellulose and concluded that $F$. succinogenes was providing cellodextrins to the other species (Scheifinger and Wolin 1973; Kudo et al. 1987; Wells et al. 1995). Altogether, the results of the present work on growing cells suggest that maltodextrins and/or maltodextrin-phosphate and/or X may be additional substrates of this inter-species cross-feeding. Indeed, two of the species used in the co-cultures, Selenomonas ruminantium and Streptococcus bovis, are amylolytic and thus able to use maltodextrins, while the third one (Treponema bryantii) is saccharolytic (Stewart et al. 1997).

A new compound, identified as cellobionic acid, was present in growing cells whatever the substrate, while it was not detected in resting cells (Table 2). The presence of cellobionic acid is very surprising. It is usually produced in aerobic lignocellulolytic fungi through the activity of a cellobiose dehydrogenase $(\mathrm{CDH})$ that converts cellobiose into cellobionolactone, an unstable compound rapidly spontaneously hydrolysed into cellobionic acid (Higham et al. 1994; Zamocky et al. 2006). CDH activity was also found in cellulolytic bacteria ( $\mathrm{Li}$ et al. 1996). Cellobionolactone was also identified in the culture media of $F$. succinogenes S85. Sugar dehydrogenase with a broad specificity is found in bacteria; for example, the 
soluble aldose sugar dehydrogenase (sGdh) from E. coli can utilise glucose or arabinose, but displays higher activity towards oligomeric substrates such as maltose, maltotriose or cellobiose (Southall et al. 2006). Putative sGdh homologs have been identified in diverse prokaryotic phyla, including the proteobacteria and some firmicutes, and even in Archaea (Oubrie 2003). The precise role of the sugar dehydrogenases is not completely clear. In aerobic fungi, $\mathrm{CDH}$ might be involved in regulation of cellulase expression as it produces cellobionolactone, which is a potent inducer of cellulase genes in some of these organisms such as Trichoderma reesei (Ilmen et al. 1997). CDH was also proposed to directly participate in cellulose depolymerisation (Mansfield et al. 1997). The E. coli as well as other enteric bacterial sGdh was proposed to be part of a periplasmic electron transfer system that can serve to input sugar-derived electrons into the respiratory network (Southall et al. 2006). In F. succinogenes, there is no clear evidence for the presence of a Gdh (no highly homologous gene was found in the genome of the S85 strain, http://blast.jcvi.org/rumenomics/), and additional experiments are necessary to discover the mechanism of cellobionolactone/cellobionic acid formation as well as its role in the bacterium.

\section{References}

Béra-Maillet C, Gaudet G, Forano E (2000) Endoglucanase activity and relative expression of glycosyl-hydrolase genes of Fibrobacter succinogenes S85 grown on different substrates. Biochim Biophys Acta 1543:77-85

Desvaux M, Guedon E, Petitdemange H (2000) Cellulose catabolism by Clostridium cellulolyticum growing in batch culture on defined medium. Appl Environ Microbiol 66:2461-2470

Desvaux M, Guedon E, Petitdemange H (2001) Metabolic flux in cellulose batch and cellulose-fed continuous cultures of Clostridium cellulolyticum in response to acidic environment. Microbiology 147:1461-1474

Fields MW, Mallik S, Russell JB (2000) Fibrobacter succinogenes S85 ferments ball-milled cellulose as fast as cellobiose until cellulose surface area is limiting. Appl Microbiol Biotechnol 54:570-574

Forano E, Delort AM, Matulova M (2008) Carbohydrate metabolism in Fibrobacter succinogenes: what NMR tells us. Microb Ecol Heath Disease 2:94-102

Gaudet G, Forano E, Dauphin G, Delort AM (1992) Futile cycling of glycogen in Fibrobacter succinogenes as shown by in situ ${ }^{1} \mathrm{H}-\mathrm{NMR}$ and ${ }^{13} \mathrm{C}-\mathrm{NMR}$ investigation. Eur J Biochem 207:155-162

Higham CW, Gordon-Smith D, Dempsey CE, Wood PM (1994) Direct ${ }^{1} \mathrm{H}$ evidence for conversion of $\beta$-D-cellobiose to cellobionolactone by cellobiose dehydrogenase from Phanerochaete chrysosporium. FEBS Lett 351:128-132

Ilmen M, Saloheimo A, Onnela ML, Penttila ME (1997) Regulation of cellulase gene expression in the filamentous fungus Trichoderma reesei. Appl Environ Microbiol 63:1298-1306

Krause DO, Denman SE, Mackie RI, Morrison M, Rae AL, Attwood GT, McSweeney CS (2003) Opportunities to improve fiber degradation in the rumen: microbiology, ecology, and genomics. FEMS Microbiol Rev 27:663-693

Kudo H, Cheng KJ, Costerton JW (1987) Interactions between Treponema bryantii and cellulolytic bacteria in the in vitro degradation of straw cellulose. Can J Microbiol 33:244-248

Li X, Huang Y, Xu D, Xiao D, Jin F, Gao P (1996) Cellobiose-oxidizing enzyme from a newly isolated cellulolytic bacterium Cytophaga sp LX-7. Biotechnol Lett 18:205-210

Lynd LR, Weimer PJ, van Zyl WH, Pretorius IS (2002) Microbial cellulose utilization: fundamentals and biotechnology. Microbiol Mol Biol Rev 66:506-577

Mansfield SD, Jong E, Sadler JN (1997) Cellobiose dehydrogenase, an active agent in cellulose depolymerisation. Appl Environ Microbiol 63:3804-3809

Matheron C, Delort AM, Gaudet G, Forano E (1997) Re-investigation of glucose metabolism in Fibrobacter succinogenes S85 using NMR and enzymatic assays. Evidence of pentose phosphates phosphoketolase and pyruvate-formate-lyase activities. Biochim Biophys Acta 1355:50-60

Matheron C, Delort AM, Gaudet G, Forano E (1998) In vivo ${ }^{13} \mathrm{C}$ NMR study of glucose and cellobiose metabolism by four cellulolytic strains of the genus Fibrobacter. Biodegradation 9:451-461

Matte A, Forsberg CW, Verinder-Gibbins AM (1992) Enzymes associated with metabolism of xylose and other pentoses by Prevotella (Bacteroides) ruminicola strains, Selenomonas ruminantium D, and Fibrobacter succinogenes S85. Can J Microbiol 38:370-376

Matulova M, Delort AM, Nouaille R, Gaudet G, Forano E (2001) Concurrent maltodextrin and cellodextrin synthesis by Fibrobacter succinogenes S85 as identified by 2D NMR spectroscopy. Eur J Biochem 268:3907-3915

Matulova M, Nouaille R, Capek P, Péan M, Forano E, Delort A-M (2005) Degradation of wheat straw by Fibrobacter succinogenes S85: a liquid and solid state NMR study. Appl Environ Microbiol 71:1247-1253

Miller T (1978) The pathway of formation of acetate and succinate from pyruvate in Bacteroides succinogenes. Arch Microbiol 117:145-152

Nouaille R, Matulova M, Delort AM, Forano E (2004) Production of maltodextrin-1-phosphate by Fibrobacter succinogenes S85. FEBS Lett 576:226-230

Nouaille R, Matulova M, Delort AM, Forano E (2005) Oligosaccharide synthesis in Fibrobacter succinogenes S85 and its modulation by the substrate. FEBS J 272:2416-2427Oubrie A (2003) Structure and mechanism of soluble glucose dehydrogenase and other PQQ-dependent enzymes. Biochim Biophys Acta 1647:143-151

Pavlostathis SG, Miller TL, Wolin MJ (1988) Fermentation of insoluble cellulose by continuous cultures of Ruminococcus albus. Appl Environ Microbiol 54:2655-2659 
Qi M, Nelson KE, Daugherty SC, Nelson WC, Hance IR, Morrison M, Forsberg CW (2005) Novel molecular features of the fibrolytic intestinal bacterium Fibrobacter intestinalis not shared with Fibrobacter succinogenes as determined by suppressive subtractive hybridization. J Bacteriol 187:3739-3751

Roger V, Fonty G, Komisarczuk-Bony S, Gouet P (1990) Effects of physicochemical factors on the adhesion to cellulose Avicel of the ruminal bacteria Ruminococcus flavefaciens and Fibrobacter succinogenes subsp. succinogenes. Appl Environ Microbiol 56:3081-3087

Scheifinger CC, Wolin MJ (1973) Propionate formation from cellulose and soluble sugars by combined cultures of Bacteroides succinogenes and Selenomonas ruminantium. Appl Microbiol 26:789-795

Stewart CS, Flint HJ, Bryant MP (1997) The rumen bacteria. In: Hobson PN, Stewart CS (eds) The rumen microbial ecosystem, Second editionth edn. Blackie, London, pp 10-72

Southall SM, Doel JJ, Richardson DJ, Oubrie A (2006) Soluble aldose sugar dehydrogenase from Escherichia coli. A highly exposed active site conferring broad substrate specificity. J Biol Chem 281:30650-30659

Van der Zee JR, Postma PW, Hellingwerf KJ (1996) Quantitative conversion of glucose into glucose-6-phosphate by intact Escherichia coli cells. Biotechnol Appl Biochem 24:225-230

Weimer PJ (1993) Effects of dilution rate and $\mathrm{pH}$ on the ruminal cellulolytic bacterium Fibrobacter succinogenes $\mathrm{S} 85$ in cellulose-fed continuous culture. Arch Microbiol 160:288-204

Wells JE, Russell JB, Shi Y, Weimer PJ (1995) Cellodextrin efflux by the cellulolytic ruminal bacterium Fibrobacter succinogenes and its potential role in the growth of non-adherent bacteria. Appl Environ Microbiol 61:1757-1762

Wells JE, Russell JB (1996) The effect of growth and starvation on the lysis of the ruminal cellulolytic bacterium Fibrobacter succinogenes. Appl Environ Microbiol 62:1342-1346

Zamocky M, Ludwig R, Peterbauer C, Hallberg BM, Divne C, Nicholls P, Haltrich D (2006) Cellobiose dehydrogenase-a flavocytochrome from wood-degrading, phytopathogenic and saprotropic fungi. Curr Protein Pept Sci 7:255-280 